\title{
CONFORMAL REFLECTIONS AND MEROMORPHIC SLIT MAPPINGS
}

\author{
URI SREBRO
}

1. In an earlier paper [3] I showed that if $f$ is a rational function in $\hat{C}=C \cup\{\infty\}$ and if $f$ maps $\Delta=\{z \in C:|z|<1\}$ injectively onto $\hat{C}-E$ where $E$ is a continuum in $\hat{C}$ with void interior, then $E$ is a circular arc or a line segment in $\hat{C}, f$ is of degree 2 and has the form

$$
f(z)=\varphi\left(\left(\frac{z-a}{z-b}\right)^{2}\right)
$$

where $a$ and $b$ are distinct points on $\partial \Delta$ and $\varphi$ is a Möbius transformation with $\varphi(0)=f(a)$ and $\varphi(\infty)=f(b)$.

As an answer to a question raised by Y. Domar about the possibility of extending this result to meromorphic functions in $C$, we now prove the following

3. Theorem. Let $f$ be a meromorphic, single-valued function in $\hat{C}-F$, where $F$ is a compact set in $\hat{C}-\bar{\Delta}$ of zero linear measure. If $f_{0}=f \mid \Delta$ maps $\Delta$ injectively onto a dense set in $\boldsymbol{C}$, then

(i) $E=\hat{C}-f_{0}(\Delta)$ is a circular arc or a line segment in $\hat{C}$.

(ii) The function $f$ is the restriction of a rational function of degree 2 and has the form (2) with distinct points, $a, b \in \partial \Delta$.

The proof of the theorem is based on a lemma about conformal reflections; see Sections 5 and 6 below.

4. An immediate corollary of the theorem applies to slit functions in the class $S$ of all univalent functions $f(z)=z+a_{2} z^{2}+\ldots$ in $\Delta$. A function $f \in S$ is called a slit function if $\Gamma=\hat{C}-f(\Delta)$ is a Jordan arc in $\hat{C}$ with a tip at $\infty$. In the following sections $\Lambda_{1}(F)$ denotes the linear measure of $F$.

Corollary. Let $f$ be a slit function in $S$. If $f$ has a single valued meromorphic continuation on $\hat{C}-F$ where $F$ is a compact set in $\hat{C}-\bar{\Delta}$ with $\Lambda_{1}(F)=0$, then the slit of $f$ is straight and $f=\varphi \circ k \circ \psi$ where $k(z)=z(1-z)^{-2}$ is the Koebe function and $\varphi$ and $\psi$ are Möbius transformations.

5. Conformal reflections and a counterexample. The assumption in the theorem and in the corollary that $f$ or its continuation is single-valued is indispensible as can 
be seen from examples which we will construct here by using the generalized reflection principle. According to this principle, cf. [2, p. 187], a continuous function $f: \bar{\Delta} \rightarrow \hat{\boldsymbol{C}}$, which is meromorphic in $\Delta$ can be continued meromorphically across every point of $\partial \Delta$, provided that there is a conformal reflection with respect to $f(\partial \Delta)$. A function $\varphi$ is said to be a conformal reflection with respect to an infinite closed set $E$ in $\hat{C}$, if $\varphi$ is antimeromorphic in some domain containing $E$ and $\varphi(z)=z$ for all $z \in E$. Obviously, not every set admits a conformal reflection and if an infinite closed set $E$ has a conformal reflection, then the conformal reflection is unique. One can show by considering $\varphi \circ \varphi$ that $\varphi$ is a (single-valued) involution in some domain containing $E$.

We now turn to the construction of a (multivalued) function $f$ (mentioned above) which is meromorphic everywhere in $\hat{\boldsymbol{C}}$ except for a finite set of points, such that $f$ maps $\Delta$ conformally onto $\hat{C}-\Gamma$ where $\Gamma$ is a compact simple arc in $C$ which is neither a line segment nor a circular arc. In this example $\Gamma$ is part of an algebraic curve.

Let $F(u, v)$ be a (real) polynomial in $u$ and $v$ and $\Gamma$ a compact simple arc in $C$, which satisfies the equation $F(u, v)=0$ and the condition $\left|F_{u}(u, v)\right|+\left|F_{v}(u, v)\right| \neq 0$ for all $(u, v) \in \Gamma$. By setting $u=(1 / 2)(w+\bar{w})$ and $v=(1 / 2)(w-\bar{w}), F(u, v)$ reduces to a polynomial $G(w, \bar{w})$ and since $G_{\bar{w}}=(1 / 2)\left(F_{u}+i F_{v}\right) \neq 0$ on $\Gamma$, it follows that $G(w, \bar{w})=0$ can be solved for $\bar{w}$ yielding a meromorphic function $g$ which is locally univalent at every point of $\Gamma$ and such that $g(w)=\bar{w}$ for all $w \in \Gamma$. Evidently, the function $\varphi=\bar{g}$ is a conformal reflection in $\Gamma$. Note that $g$ is algebraic and hence it is meromorphic everywhere except for a finite set in $\hat{\boldsymbol{C}}-\Gamma$.

Now let $f$ be a conformal mapping of $\Delta$ onto $\hat{C}-\Gamma$. Then $f$ can be continued by letting $f(z)=\varphi(f(1 / \bar{z}))$ for $z \in \hat{\boldsymbol{C}}-\Delta$. Since $\bar{\varphi}$ is algebraic, it follows that $f$ is meromorphic everywhere in $\hat{\boldsymbol{C}}$ except for a finite set. It is clear that in the above construction $\Gamma$ need not be a line segment of a circular arc.

6. Lemma. Let $\varphi$ be a conformal reflection with respect to an infinite closed set $E$. If $\varphi$ is single-valued (antimeromorphic) in $D=\hat{C}-F$, where $F$ is a compact set in $C-E$ with $\Lambda_{1}(F)=0$, then

(i) $E$ is contained in a straight line or in a circle.

(ii) The function $\varphi$ is injective and $\bar{\varphi}$ is the restriction of a Möbius transformation.

Proof of the lemma. $\Lambda_{1}(F)=0$ and $\varphi$ is meromorphic, hence by using injective branches of $\varphi^{-1}$ one sees that $\Lambda_{1}\left(\varphi^{-1}(F)\right)=0$. Consequently, $\Lambda_{1}\left(F \cup \varphi^{-1}(F)\right)=0$ and $D_{0}=D-\varphi^{-1}(F)$ is a domain. Let $\varphi_{0}=\varphi \mid D_{0}$. Then $h=\varphi \circ \varphi_{0}$ is a well defined single-valued meromorphic function in $D_{0}$. Now, $\varphi(w)=w$ for all $w \in E$, hence $h(w)=w$ for all $w \in E$, and since $E$ is infinite and closed in $\hat{\boldsymbol{C}}$, it follows that $E$ clusters in the domain of $h$ and thus $h(w)=w$ for all $w \in D_{0}$. Consequently, $\varphi_{0}$ is injective in $D_{0}$. Indeed, $\varphi\left(w_{1}\right)=\varphi\left(w_{2}\right)$ for $w_{1}, w_{2} \in D_{0}$ implies

$$
w_{1}=\varphi\left(\varphi\left(w_{1}\right)\right)=\varphi\left(\varphi\left(w_{2}\right)\right)=w_{2} .
$$


Next $\Lambda_{1}\left(F \cup \varphi^{-1}(F)\right)=0$ and $\bar{\varphi}_{0}$ is conformal, hence, by a known removability theorem (cf. [1]) applied to $\bar{\varphi}_{0}$ it follows that $\bar{\varphi}_{0}$ has a conformal extension on $\hat{\boldsymbol{C}}$ and thus (ii) follows.

Finally, note that the fixed set of an anti-Möbius transformation is either finite or a circle or a line, thus (i) follows by (ii) and the fact that $\varphi$ fixes every point of $E$.

7. Proof of the theorem. We first show that $E$ admits a conformal reflection which satisfies the assumptions of the lemma. This will imply part (i) of the theorem.

Let $\psi(z)=1 / \bar{z}$ and $F^{\prime}=f_{0} \circ \psi(F)$. Evidently, $F^{\prime}$ is compact and $F^{\prime} \cap E=\emptyset$, and since $f_{0} \circ \psi$ is a diffeomorphism in $\hat{C}-\bar{\Delta}$ and $\Lambda_{1}(F)=0$, it follows that $\Lambda_{1}\left(F^{\prime}\right)=0$. Let $f_{1}=f \mid \hat{C}-\Delta-F$, then

$$
\varphi=f_{1} \circ \psi \circ\left(f_{0}^{-1} \mid \hat{\boldsymbol{C}}-E-F^{\prime}\right)
$$

is antimeromorphic in $\hat{\boldsymbol{C}}-E-F^{\prime}$. Now, $f$ has only finitely many branch points on $\partial \Delta, f_{0}$ is a homeomorphism and int $E=\emptyset$, therefore

$$
E=\partial f_{0}(\Delta)=f(\partial \Delta)
$$

is a finite union of analytic arcs. Furthermore, $\varphi$ has a continuous extension on $\hat{\boldsymbol{C}}-F^{\prime}$ denoted again by $\varphi$, with $\varphi(w)=w$ for all $w \in E$. Hence, by applying Morera's theorem to $\bar{\varphi}$, or by [2, p. 183] it follows that $\varphi$ is antimeromorphic in $\hat{\boldsymbol{C}}-F^{\prime}$. Therefore, $\varphi$ is a conformal reflection in $E$ and satisfies the assumptions of the lemma. Thus, by part (i) of the lemma, $E$ is either a circular arc or a line segment in $\hat{C}$ as stated in part (i) of the theorem.

We now turn to part (ii) of the theorem. Since $E=f(\partial \Delta)$ is either a circular arc or a line segment in $\hat{\boldsymbol{C}}$, we may apply the standard reflection principle to $f_{0}$. By doing so noting that $f_{0}$ is univalent, we conclude that $f$ is a rational function of degree two and that $f$ maps its two branch points to the two tips of $E$. By using an auxiliary Möbius transformation $\varphi$ which maps 0 and $\infty$ to the tips of $E$ it is not hard to verify that $f$ has the form (2) ( $c f$. [3]). This completes the proof of the theorem.

8. Acknowledgement. I wish to thank Yngve Domar for stimulating questions, the Academy of Finland for a research grant which I obtained during a visit to Finland. The research was partially supported by the fund for the promotion of research at the Technion. 


\section{References}

[1] Besicovitch, A. S.: On sufficient conditions for a function to be analytic, and on behaviour of analytic functions in the neighbourhood of non-isolated singular points. - Proc. London Math. Soc. (2) 32, 1931, 1-9.

[2] Nehari, Z.: Conformal mapping. - McGraw-Hill Book Company, Inc., New York-Toronto -London, 1952.

[3] SRebro, U.: Is the slit of rational slit mappings in $S$ straight? - Proc. Amer. Math. Soc. (to appear).

\section{Technion}

Department of Mathematics

Haifa 32000

Israel

Received 1 August 1983 